\title{
Keanekaragaman Burung di Sungai Mamberamo, Papua
}

\author{
Birds Diversity at the Mamberamo River Basin, Papua
}

\author{
Freddy Pattiselanno
}

Jurusan Produksi Ternak Fakultas Peternakan Perikanan \& Ilmu Kelautan

Universitas Negeri Papua E-mail: fpattiselanno@yahoo.com

The Mamberamo-Rouffaer-Idenburgh River watershed, one of the wetland sites in West Papua is the largest tropical marshlands in northern Papua, covers 7,711,602 hectares and includes in the North Papuan Lowlands Endemic Bird Area (EBA). The Irian Jaya Biodiversity Conservation Priority-Setting Workshop (Conservation International, 1999) highlighted that the Mamberamo Basin is an area with high priority for conservation. It is also acknowledged little biological information has been collected therefore it is urgent to carry out biological and ecological study in this particular sites.

Conservation International has conducted a Rapid Biodiversity Assessment Program to collect biological information from Mamberamo, and it was noted that approximately 143 species of birds were recorded among the lowland forest, swamp, and riverine sites near the village of Dabra (Van Balen et al., 2002). This paper presents results of current survey that accomplished in connection with the Natural Resources Mapping Program, a collaboration project between Pemda Kabupaten Sarmi and Universitas Negeri Papua.

Survey was achieved from 4 - 28 September 2004 in the Dabra $\left(03^{\circ} 16^{\prime} \mathrm{S}\right.$ $\left.138^{0} 36^{\prime} \mathrm{E}\right)$, Taiyeve (03 $\left.{ }^{0} 14.06^{\prime} \mathrm{S} 138^{0} 26.62^{\prime} \mathrm{E}\right)$ and Fokri-Baso $\left(03^{0} 05.08^{\prime} \mathrm{S}\right.$ 138 $\left.50.12^{\prime} \mathrm{E}\right)$ villages of the Mamberamo Hulu District of Kabupaten Sarmi, Papua. Bird's observation was conducted from 0530 to 0930 hours during daytime and 1600 to 1800 at nighttime, along boat-transect line at the Mamberamo River from Dabra to Taiyeve \pm $\mathrm{km}$ from 0800 hours to 1200 hours, and from Dabra to Fokri-Baso $\pm \mathrm{km} 0730$ hours to 1400 hours. Birds were observed with the aid of Burris binocular $10 \mathrm{X}-50 \mathrm{~mm}$ and identification was performed by using the taxonomic description from the book "Handbook of New Guinea Birds (Rand and Gilliard, 1967) and "Birds of New Guinea (Beehler et al., 1986).

A complete list from the survey is presented in Table 1, compared with previous studies achieved at the same relative location in the study site.

Tabel 1. Birds species found along Mamberamo River Basin, Papua

\begin{tabular}{|c|c|c|c|c|}
\hline Species & Vernacular Name & CI (2002) & Archbold (1938-39) & Remarks \\
\hline Accipiter cirrchocephalus & " Collared Sparrow Hawk & $\sqrt{ }$ & & \\
\hline Accipiter poliocephalus & Grey-headed Goshawk & $\sqrt{ }$ & & E-NG \\
\hline Actitis hypoleucos & Common Sandpiper & $\sqrt{ }$ & & \\
\hline Aerodramus vanikorensis & Lowland Swiftlet & $\sqrt{ }$ & $\sqrt{ }$ & \\
\hline Alcedo azurea & Azure Kingfisher & $\sqrt{ }$ & $\sqrt{ }$ & \\
\hline Ardea sumatrana & Giant Heron & $\sqrt{ }$ & $\mathrm{U}$ & \\
\hline Arses telescophthalmus & Frilled Flycatcher & $\sqrt{ }$ & $\sqrt{ }$ & \\
\hline Artamus leucorynchus & Lesser Wood Swallow & $\sqrt{ }$ & $\mathrm{F}$ & \\
\hline Aviceda subcristata & Crested Lizard Hawk & $\sqrt{ }$ & $\mathrm{U}$ & \\
\hline Butorides striatus & Mangrove Heron & $\sqrt{ }$ & $\mathrm{F}$ & \\
\hline Cacatua galerita & White Cockatoo & $\sqrt{ }$ & $\mathrm{C}$ & \\
\hline Cacomantis variolosus & Brush Cuckoo & $\sqrt{ }$ & $\sqrt{ }$ & \\
\hline Casuarius unappendiculatus & Single-wattled Cassowary & $\sqrt{ }$ & $\sqrt{ }$ & E-NG \\
\hline Cicinnurus regius & King Bird of Paradise & $\sqrt{ }$ & $\sqrt{ }$ & E-NG \\
\hline Coracina schisticeps & Gray’s Greybird & $\sqrt{ }$ & $\sqrt{ }$ & E-NG \\
\hline Coracina papuensis & Papuan Greybird & $\sqrt{ }$ & $\mathrm{F}$ & \\
\hline
\end{tabular}


Tabel 1. Continued

\begin{tabular}{|c|c|c|c|c|}
\hline Species & Vernacular Name & CI (2002) & Archbold (1938-39) & Remarks \\
\hline Corvus tristis & Grey Crow & $\sqrt{ }$ & $\sqrt{ }$ & E-NG \\
\hline Dacelo gaudichaud & $\begin{array}{l}\text { Rufous-bellied Giant } \\
\text { Kingfisher }\end{array}$ & $\sqrt{ }$ & $\sqrt{ }$ & E-NG \\
\hline Dicaeum pectorale & Olive-crowned Flowerpecker & $\sqrt{ }$ & & E-NG \\
\hline Egretta garzetta & Snowy Egret / Little Egret & $\sqrt{ }$ & $\mathrm{C}$ & \\
\hline Egretta intermedium & Intermedium Egret & $\sqrt{ }$ & $\mathrm{C}$ & \\
\hline Egretta picata & Pied Heron & $\sqrt{ }$ & $\mathrm{U}$ & \\
\hline Eurystomus orientalis & Broad-billed Roller & $\sqrt{ }$ & $\sqrt{ }$ & \\
\hline Geoffroyus geoffroyi & Red-cheeked Parrot & $\sqrt{ }$ & $\mathrm{C}$ & \\
\hline Gerygone magnirostris & Swamp Gerygone Warbler & $\sqrt{ }$ & $\mathrm{C}$ & \\
\hline Goura Victoria & Victoria Crowned Pigeon & $\sqrt{ }$ & $\sqrt{ }$ & E-NG \\
\hline Haliaeetus leucogaster & White-bellied Sea Eagle & $\sqrt{ }$ & $\mathrm{F}$ & \\
\hline Haliastur Indus & Brahminy Kite & $\sqrt{ }$ & $\mathrm{C}$ & \\
\hline Halycon torotoro & $\begin{array}{l}\text { Lesser Yellow-bellied } \\
\text { Kingfisher }\end{array}$ & $\sqrt{ }$ & & \\
\hline Himantopus leucocephalus & White-headed Stilt & $\sqrt{ }$ & $\mathrm{R}$ & \\
\hline Hirundo rustica & European Swallow & $\sqrt{ }$ & & \\
\hline Lorius lorry & Western Black-capped Lory & $\sqrt{ }$ & $\mathrm{F}$ & E-NG \\
\hline Malurus alboscapulatus & $\begin{array}{l}\text { Black and White Wren } \\
\text { Warbler }\end{array}$ & $\sqrt{ }$ & $\mathrm{C}$ & E-NG \\
\hline Megapodius freycinet & Common Scrub Hen & $\sqrt{ }$ & $\sqrt{ }$ & \\
\hline Melilestes megarhynchus & Long-beaked Honeyeater & $\sqrt{ }$ & $\sqrt{ }$ & E-NG \\
\hline Melipagha aruensis & Mimic Melipagha & $\sqrt{ }$ & $\sqrt{ }$ & \\
\hline Mino dumontii & Yellow-faced Mina & $\sqrt{ }$ & $\mathrm{F}$ & \\
\hline Monarcha guttula & Spot-wing Monarch & $\sqrt{ }$ & $\sqrt{ }$ & \\
\hline Nectarinia aspasia & Yellow-bellied Sunbird & $\sqrt{ }$ & $\sqrt{ }$ & \\
\hline Ninox theomacha & Brown Owl & $\sqrt{ }$ & & E-NG \\
\hline Oriolus szalayi & Brown Oriole & $\sqrt{ }$ & $\mathrm{F}$ & E-NG \\
\hline Paradisea minor & Lesser Bird of Paradise & $\sqrt{ }$ & $\sqrt{ }$ & E-NG \\
\hline Phalacrocorax melanoleucos & Little Pied Cormorant & $\sqrt{ }$ & $\sqrt{ }$ & \\
\hline Phalacrocorax sulcirostris & Little Black Cormorant & $\sqrt{ }$ & $\sqrt{ }$ & \\
\hline Philemon novaeguinae & New Guinea Friar Bird & $\sqrt{ }$ & $\sqrt{ }$ & \\
\hline Podargus papuensis & Great Papuan Frogmouth & $\sqrt{ }$ & & \\
\hline Probosciger aterrimus & Palm Cockatoo & $\sqrt{ }$ & $\sqrt{ }$ & \\
\hline Psittaculirostris salvadorii & Salvadori's Fig Parrot & $\sqrt{ }$ & $\mathrm{R}$ & E-NG \\
\hline Psittrichas fulgidus & Vulturine Parrot & $\sqrt{ }$ & $\sqrt{ }$ & E-NG \\
\hline Ptilinopus superbus & Superb Fruit-Dove & $\sqrt{ }$ & $\sqrt{ }$ & \\
\hline Rhipidura rufiventris & White-throated Fantail & $\sqrt{ }$ & $\sqrt{ }$ & \\
\hline Tadorna radjah & White-headed Shelduck & $\sqrt{ }$ & & \\
\hline Talegalla jobiensis & Brown-collared Brush Turkey & $\sqrt{ }$ & $\sqrt{ }$ & E-NG \\
\hline
\end{tabular}

Note: $\sqrt{ }$ - present, $\mathrm{C}$ - common, $\mathrm{F}$ - fairly common, $\mathrm{U}$ - uncommon, $\mathrm{R}$ - rare, E-NG - Endemic New Guinea

Fifty-three species were recorded during the survey or about $37 \%$ compared to the previous study by Conservation International (Van Balen et al., 2002) and all species seen in this survey were also recorded during the RAP. Seventeen species from 53 birds or $32 \%$ from a total species found during this study were endemic to New Guinea. Some species (Cacatua galerita, Casuarius unappendiculatus, Goura victoria and Lorius lorry) were domesticated and raised as pets by local people in Dabra (Pattiselanno dan Metelmety, 2004).

\section{Literature Cited}

Archbold, R., Rand, A.L. and Brass, J.L. 1942. Results of the Archbold Expeditions. No. 41. Summary of the 1938-1939 New Guinea Expedition. Bull. Am. Mus. Nat. Hist. 79: 197-288. 
Beehler, B., Pratt, T.K. and Zimmerman, D.A. 1986. Birds of New Guinea. New Jersey: Princeton University Press. 293 pp

Conservation International. (ed.) 1999. The Irian Jaya biodiversity conservation priority-setting workshop. Final Report. Washington, DC: Conservation International.

Pattiselanno, F. dan Metelmety, R. 2004. Laporan Studi Pembuatan Atlas Sumberdaya Kabupaten Sarmi (Distrik Mamberamo Hulu). Universitas Negeri Papua, Manokwari.

Rand, A.L. and Gilliard, E.T. 1967. Handbook of New Guinea Birds. The Trinity Press, Worcester, London. $612 \mathrm{pp}$

Van Balen, B., Suryadi, S. and Kalo, D. 2002. Birds of the Dabra Area, Mamberamo River Basin, Papua, Indonesia. In Richards, S.J. and Suryadi, S. (editors). A Biodiversity Assessment of Yongsu - Cyclops Mountains and the Southern Mamberamo Basin, Papua, Indonesia. RAP Bulletin of Biological Assessment 25. Conservation International, Washington, DC, USA: 8488. 